\title{
Kiswahili Katika Taathira ya Kihistoria na Mielekeo ya Kisiasa kama Lugha ya Kufundishia
}

\section{Gerephace Mwangosi}

Idara ya Lugha, Chuo Kikuu cha Kikatoliki Ruaha (RUCU)

\section{IKISIRI}

Makala hii inahusu Kiswahili katika taathira ya kihistoria na mielekeo ya kisiasa kama lugha ya kufundishia elimu ya juu hapa nchini Tanzania. Makala hii inaeleza mambo yanayosababisha mielekeo tofauti ya watu kuhusu upokezi na matumizi ya lugha ya Kiswahili kama lugha ya kufundishia elimu ya juu nchini Tanzania ili kushurutisha ukubalifu wake. Baadhi ya masuala yanayochunguzwa ni yale yanayotiliwa shaka kuhusu uwezo wa lugha ya Kiswahili kumudu na kukidhi harakati za mahitaji ya kufundishia elimu ya juu, pamoja na kupitisha teknolojia mpya inayoinukia katika nyanja za sayansi na teknolojia. Aidha, makala hii inanuia kufafanua ukubalifu, pamoja na msingi wa mielekeo ya Watanzania kutoipatia kipaumbele lugha ya Kiswahili kama lugha inayoweza kukidhi mahitaji ya kufundishia elimu ya juu hapa nchini. Data za msingi katika makala hii zilipatikana maktabani kwa kudurusu nyaraka mbalimbali zilizohusiana na mada iliyolengwa. Mapitio ya nyaraka mbalimbali zilizotumika kushadidia data za msingi za mada iliyochunguzwa zilipatikana katika maktaba ya chuo kikuu cha Kikatoliki cha Ruaha iliyopo Iringa. Makala hii inahitimisha kwamba lugha za kigeni na zile za kiasili zinaweza kuendelea kutumika pamoja katika ngazi zote za elimu ili kuziwezesha kutekeleza vema dhima zake.

Historia ya Makala

Kupokelewa: 10 Aprili 2018

Kukubaliwa 11

Novemba 2018

\section{Istilahi Muhimu}

Taathira; Taathira ya kihistoria; Mielekeo ya kisiasa; Lugha ya kufundishia

\section{Jinsi ya kurejea Makala hii:}

Mwangosi, G. 2018. Kiswahili Katika Taathira ya Kihistoria na Mielekeo ya Kisiasa kama Lugha ya Kufundishia. Mkwawa Journal of Education and Development, 2, 3448. DOI: https://doi.org/10.37759/mjed.2018.2.1.3

\begin{tabular}{ll}
\hline Anuani: & $\begin{array}{l}\text { Chuo Kikuu cha Kikatoliki } \\
\text { mwangosigerephace@yahoo.com }\end{array}$
\end{tabular}




\subsection{Utangulizi}

Lugha ni zao la utamaduni na kila lugha ina mwenendo na kanuni zake. Maendeleo katika historia ya mitazamo na itikadi yanathibitisha kwamba, kuna uhusiano mkubwa baina ya lugha na wazo, na baina ya wazo na tajriba (Mhina, 1977; Mekacha, 2000). Kwa mtazamo huo, kujaribu kutafsiri mambo au mawazo kwa lugha nyingine huweza kuathiri au kusambaratisha mitazamo iliyojengwa kupitia lugha za asili za jamii. Mbali na kuwasiliana, lugha hutazamwa kama benki jumuishi ya fikra na kumbukumbu za tajriba ya watu katika historia; na kama maktaba kuu iliyosheheni kaida na utamaduni wa kila jamii hapa duniani (Ngugi, 1986). Kwa msingi huo, lugha ni kifaa kinachoweza kutumiwa kurithisha maarifa, historia, mila, desturi, tajriba, falsafa na mitazamo ya jamii.

Aidha, suala la lugha fulani kupata hadhi ya kufundishia elimu halihitaji ushabiki wowote, ukiwamo wa kisiasa au kijiografia. Lugha yoyote inapotajwa kuwa ina hadhi ya kufundishia, vipo vigezo mahsusi vinavyochunguzwa. Baadhi ya vigezo vya lugha inayofaa kufundishia elimu ni ile ya kutonasibishwa na watu au kabila maalumu pamoja na kuwapo kwa idadi kubwa ya watumiaji. Sera ya lugha ya nchi yoyote inayolenga kukidhi haja ya mamlaka inaweza kuundwa kutegemea na hali halisi iliyopo kuhusu lugha inayolengwa (Kalondero, 1983; Msanjila, 1979). Kwa hiyo, makala hii inahusu Kiswahili katika taathira ya kihistoria na mielekeo ya kisiasa kama lugha ya kufundishia elimu ya juu hapa nchini.

\section{2.o Mada Iliyochunguzwa}

Suala la nafasi ya Kiswahili kama lugha ya kufundishia elimu hapa nchini limeshughulikiwa na wanazuoni wengi kwa mitazamo na misukumo mbalimbali. Licha ya kuwapo kwa jitihada mbalimbali zilizokwishafanywa na baadhi ya wataalamu hao kwa mfano, (Mhina, 1977; Rubagumya, 1986; Msanjila, 1990; Kiango, 1999; Mulokozi, 2000; Qorro, 2000) kuhusu lugha ya Kiswahili kwa ujumla wake, kwa kadiri ya ufahamu wetu, bado utafiti kuhusu Kiswahili katika taathira ya kihistoria na mielekeo ya kisiasa kama lugha ya kufundishia hapa nchini haujafanywa kwa kiwango cha kutosha. Makala hii imeshughulikia suala hilo kwa kuchunguza Kiswahili katika taathira ya kihistoria na mielekeo ya kisiasa kama lugha ya kufundishia elimu ya juu hapa nchini. 


\section{3.o Yaliyoandikwa Kuhusu Lugha ya Kufundishia}

Kiswahili katika taathira ya kihistoria

Lugha ya kufundishia ni mojawapo ya sifa muhimu na zenye athari kubwa katika mfumo wowote wa elimu katika taifa lolote. Lugha ya kufundishia ndiyo inayotumika kujenga mfumo wa elimu, kubuni na kuendeleza maarifa, pamoja na kulimbikiza na kuhifadhi maarifa (Giles, 1982; Massamba, 1993). Kimsingi, lugha ya kufundishia haithibitishi tu uwezo wa lugha hiyo kuendelea na kukua kama lugha ya utamaduni, sayansi na teknolojia, bali pia huwezesha watu kujiamini wakiwa wamesimama katika misingi ya historia, falsafa na utamaduni wao. Lugha kama hiyo huwajengea watu uwezo na nguvu za kiutamaduni, kisayansi na kiteknolojia katika taifa lao (Msanjila, 2009).

Kwa kawaida, katika jamii huru, uhawilishaji wa maarifa hufanywa kwa kutumia lugha za jamii zinazotumiwa na umma kufikiri na kubuni mambo yao (Prah, 2003). Hii ina maana kwamba, katika taifa lolote, uhuru wa kiutamaduni hauwezi kukuzwa, kupanuliwa na kuendelezwa ikiwa lugha ya kufundishia ni tofauti na lugha inayotumiwa na watu katika maisha yao ya kawaida ya kila siku. Hali hii ndiyo iliyotamalaki katika nchi za Afrika Mashariki, ambapo, lugha zake, bado zimekosa ithibati inayotekelezeka kuwa lugha ya kufundishia katika ngazi zote za elimu. Suala la umuhimu wa kutumia lugha yoyote ya kwanza kufundishia elimu katika jamii za Kiafrika linapewa uzito na Kiango (1999) anaposema:

Lugha za jamii ni bora katika kufundishia kuliko zile za kigeni, kwa sababu, kwanza, kisaikolojia lugha ya jamii ni mfumo wa alama zenye maana ambazo katika akili ya mwanafunzi hufanya kazi haraka katika kujieleza na kuelewa. Pili, kisaikolojia, lugha ya mtu ni njia ya kujinasibisha na jamii yake. Tatu, kielimu, mwanafunzi hujifunza haraka kupitia lugha anayoielewa vema kuliko lugha ngeni.

Kwa mujibu wa dondoo hilo, lugha ya kufundishia inapokuwa tofauti na lugha ya jamii, wale wanaotumia lugha ya kigeni hushindwa kujinasibisha na wanajamii wenzao. Kwa kawaida, katika mazingira haya huwa kuna historia ya kuwapo kutawaliwa au ukoloni baina ya taifa moja na jingine. Katika kuipa nguvu hoja hii, Mammino (2000, uk. 23) anaeleza:

Matumizi ya lugha ya pili kama lugha ya kufundishia ni urithi wa kikoloni. Katika nchi zote ambako urithi huu haupo, wanafunzi hutumia lugha zao za kwanza kwa muda wote wa kupata elimu. Kwa mtazamo wa kiufundishaji, matumizi ya lugha ya pili huathiri wepesi na maliwazo ya wanafunzi kupata maarifa kwa upana na kwa kina. 
Nukuu hiyo inasisitiza kuwapo kwa umuhimu wa kutumia lugha ya kwanza kufundishia elimu. Hali hiyo huwawezesha wanajamii kuendeleza mambo yao kwa misingi ya mila na desturi zao, pamoja na kuwajengea uwezo wa kuwa huru kifikra na kuimarisha umoja na mshikamano wao. Aidha, maarifa yanaweza kubuniwa kupitia lugha yao ya kwanza kwa kuendelezwa na wahusika moja kwa moja hadi kufikia upeo wake wa kimantiki na kifalsafa. Hivyo, utoaji wa elimu unapaswa kutilia maanani misingi ya utekelezaji wake ambao kwa kiasi kikubwa, hufanikishwa kwa njia ya matumizi ya lugha inayoeleweka vizuri. Akionesha urahisi wa kutumia lugha ya kwanza kufundishia, Mbaabu (2009, uk. 97) anaeleza:

Huku inapomchukua kijana hodari Mwafrika muda wa miezi sita tu kujifunza lugha ili aweze kukisoma kitabu cha Kiswahili na kukielewa barabara, ingemchumkua angalau miaka sita ya kujifunza Kiingereza kwa makini ili aweze kukielewa kitabu cha Kiingereza cha kiwango sawa. Ni vigumu kuelewa vile watetezi wa Kiingereza wanavyotarajia kustawisha elimu ya Waafrika kwa kutumia lugha ya Ulaya kama lugha ya kufundishia masomo mengine.

Maelezo haya ni ya msingi sana kuhusu hadhi ya lugha za kwanza barani Afrika kupewa nafasi ya kufundishia katika ngazi zote za elimu. Mtazamo wa Mbaabu unaitazama lugha ya Kiswahili katika jamii za Afrika Mashariki kuwa ina hadhi ya kukuza fikra pevu ambazo ni msingi wa ubunifu na ugunduzi. Licha ya kuwa lugha ya Kiswahili imeendelea kukua na kukomaa kama lugha ya taifa na rasmi katika baadhi ya mataifa ya Afrika Mashariki, bado lugha ya Kiingereza imepewa hadhi ya kuwa lugha ya kufundishia katika ngazi ya elimu ya juu katika fani za sheria, uhandisi, utabibu (Buliba na Wenzake, 2006). Hali hii inatoa mwanya unaoweza kuathiri mfumo wa maarifa yanayoweza kubuniwa kupitia lugha ya kwanza yanayoweza kuendelezwa na wahusika hadi kufikia upeo wake wa kimantiki na kifalsafa. Kwa msingi huo, kuhusu lugha ya Kiswahili, ni wakati muafaka wa kuunda sera ya lugha inayotekelezeka kwa kuhusisha nchi zote za Afrika Mashariki. 


\section{4.o Kiswahili Kufundishia Elimu ya Juu}

Katika ngazi hii ya elimu, kama ilivyo katika ngazi nyingine, lugha ya kufundishia ingefaa iwe lugha ya kwanza ${ }^{1}$ ili kuendeleza na kuimarisha misingi iliyowekwa awali. Ngazi hii ya elimu, inajishughulisha na maudhui mapana na ya kina ya maarifa. Hali hii imewafanya watu wengi, hasa wasomi na wanasiasa katika mataifa ya Kiafrika waamini kuwa maarifa kama hayo hayawezi kuwasilishwa kwa lugha ya kwanza, isipokuwa kwa lugha za kigeni (Mazrui, 1995; Mwakyembe, 2003). Msingi wa hoja hii, kwa kiasi fulani, ni kweli, kwa sababu ili kuwasilisha maarifa ya juu huhitajika lugha iliyoendelezwa kwa ajili hiyo; na lugha kubwa za kigeni ndizo zilizokwishaendelezwa kwa kiasi kikubwa na kuzifanya zijulikane kama lugha za elimu ya juu, sayansi na teknolojia.

Nchini Tanzania, lugha ya Kiingereza ndiyo inayotumika kama lugha ya kufundishia katika ngazi ya elimu ya juu, hasa katika fani za sayansi na teknolojia. Kulingana na Fishman (1968), takribani nchi zote za Afrika Mashariki na kwingineko barani Afrika, ngazi hii ya elimu ndiyo uwanja wa kufundishia lugha za kigeni, zikiwamo lugha za Kiingereza na Kifaransa (Fishman (1968). Kihistoria, Tume ya Phelps-Stokes iliyokuwa na mwelekeo wa kisiasa uliochukuliwa na Waafrika walioshika madaraka mara tu baada ya ukoloni, ilipendekeza kwamba, Waafrika walikuwa na haki ya kusomeshwa kwa kutumia lugha zao za kwanza ili kukuza fikra pevu ambazo ni msingi wa ubunifu na ugunduzi (Mbaabu, 2009). Ikilinganisha lugha za kwanza na Kiingereza, Tume hiyo ilisema hivi:

...thamani za lugha ya kwanza ni kubwa sana kwa sababu ndizo zinazoweza kuhifadhi itikadi njema zilizoko katika utamaduni wa Waafrika; na kwa kufanya hivyo, huhifadhi kile kinachoshinda yote kwa umuhimu, kujiheshimu na dhuluma kubwa zaidi unayoweza kuwafanyia watu ni kuwanyima lugha yao (Jones, 1925, uk. 8).

Kimsingi, lugha ya Kiswahili ni miongoni mwa lugha za kiasili zilizotamalaki katika jamii za Afrika Mashariki zenye wasemaji wengi hata nje ya mipaka ya jamiilugha yake (Ogechi, 2002). Lugha ya Kiswahili kama ilivyo kwa upande wa lugha nyingine za jamii za kimataifa, imekuwa chombo muhimu cha mawasiliano miongoni mwa nchi nyingi barani Afrika. Lugha ya Kiswahili inazungumzwa na imestawi takribani katika nchi 19

\footnotetext{
${ }^{1}$ Makala hii inaitazama lugha ya Kiswahili kuwa miongoni mwa lugha za wazawa zilizotamalaki katika jamii za Afrika Mashariki, hasa tupozungumzia lugha za kigeni kama Kiingereza kuendelea kutumika na kutazamwa kama lugha ya kufundishia elimu ya juu, sayansi na teknolojia katika baadhi ya mataifa ya Kiafrika.
} 
barani Afrika na nje yake; na hivi sasa inakadiriwa kuwa na watu zaidi ya milioni 100 wanaozungumza Kiswahili duniani (Kajenje, 2007; Marani, 2007). Pia, kuna vituo zaidi ya 100 vya redio na televisheni katika nchi mbalimbali, ikiwamo Uingereza (BBC), Ujerumani (Deutsche Welle) na Amerika (Voice of America). Kipekee, lugha ya Kiswahili haina budi kupewa jukumu la kubeba ujuzi wa kisayansi, falsafa na teknolojia za ndani na nje ya bara la Afrika, pamoja na kutumiwa kufundishia katika ngazi zote za elimu.

Pili, lugha ya kufundishia elimu haina budi kufikia hatua ya kuwekewa mikakati ya kuendelezwa. Lugha iliyoendelezwa ndiyo inayoweza kuwasilisha maarifa mapana na ya kina yanayotolewa katika ngazi ya elimu ya juu; hivi ndivyo ilivyofanyika kwa lugha kubwa za kigeni (Ansre, 1977). Kwa upande wa eneo la Afrika Mashariki, lugha ya Kiswahili ndiyo lugha pekee iliyofikia hatua ya kuwekewa mikakati ya kuendelezwa. Lugha hii imeendelezwa kwa takribani miaka 90 tangu miaka ya 1920 na 1930 hadi sasa ukichunguza historia yake ya usanifishaji (Chiraghdin \& Mathias, 1977; Mbaabu, 1991; Chachage, 2003). Kwa msingi huo, lugha hii ina historia ndefu ya kujengwa na kuendelezwa kiasi cha kufikia kuwa lugha ya taifa na rasmi iliyojengewa uwezo wa kuwasilisha maarifa mapana na ya kina katika ngazi zote za elimu.

Hoja ya kuendelezwa na kuwekewa mkakati kwa lugha ya Kiswahili ilianza, hasa ilipoteuliwa kuwa lugha rasmi ya Umoja wa Nchi Huru za Kiafrika (UNHA) mnamo mwaka 1986, ingawa utekelezaji wake rasmi ulianza julai mwaka 2004 ambapo lugha ya Kiswahili ilianza kutumika rasmi kwa mara ya kwanza katika vikao vya Umoja wa Afrika (AU). Baadhi ya maazimio yaliyopitishwa ili kurasimisha lugha ya Kiswahili yanavyodokezwa na Mwakisimba (2007). Kwanza, kuwapo kwa mpango wa Afrika wa kuendeleza lugha (1986), ambapo azimio la kumi na sita linahusu lugha ya Kiswahili kutumika katika vikao vya Umoja wa Nchi Huru za Kiafrika. Pili, Azimio la Harare (Harare Declaration), kifungu cha (4.IE) (1997) kuhusu sera ya lugha na matumizi ya Kiswahili katika vikao vya Umoja wa Nchi Huru za Kiafrika. Tatu, Azimio la Maputo la Mkutano Mkuu wa Mawaziri wa Umoja wa Afrika lililosisitiza lugha ya Kiswahili ianze kutumika katika vikao vya Umoja wa Afrika mwaka 2003.

Hapana shaka kuwa, suala la Kiswahili kama lugha ya kwanza kutumiwa kufundishia katika ngazi zote za elimu ni muhimu sana. Hapa nchini, lugha ya Kiingereza ndiyo 

inayotumika kama lugha ya kufundishia takribani katika fani zote za elimu ya juu. Aidha, matumizi ya lugha za kigeni yameathiri uimarikaji wa uelewa wake kwa wanaojifunza. Ngazi ya elimu ya juu inahitaji lugha yenye uwezo wa kuwasilisha maarifa mapana na ya kina. Kukua na kukomaa kwa lugha ya Kiwahili katika mataifa ya Afrika Mashariki, kunaifanya itazamwe kama lugha muafaka inayoweza kumudu mahitaji ya kufundishia ngazi zote za elimu.

\section{5.o Kiswahili Katika Taathira ya Kihistoria na Mitazamo ya Kisiasa na Kisera kama Lugha ya Kufundishia Elimu ya Juu}

Mitazamo kuhusu lugha, kwa upana wake, ni ukadiriaji wowote wa kihisia, kiufahamu na kitabia kuhusu mwitikio wa wazungumzaji wa lugha inayohusika (Ryan \& wenzake, 1982). Jambo linalosisitizwa katika ufafanuzi wa mitazamo ni ile hali ya kuwa tayari kiakili na kuitumia lugha katika nyanja zote za kijamii. Mitazamo ya lugha hujumuisha hisia za kupenda au kutopenda lugha fulani; pamoja na umuhimu wa kijamii unaoambatanishwa na lugha, na namna mbalimbali za matumizi ya lugha katika jamii. Kwa kawaida, lugha yenye matumizi mapana itakuwa na mtazamo chanya inapolinganishwa na lugha nyingine ndogo kimatumizi (Edwards, 1982). Hoja hii ndiyo inayoibua mitazamo chanya na hasi kuhusu matumizi ya lugha ya Kiswahili kama lugha ya kufundishia elimu ya juu.

Umuhimu wa lugha huchangiwa pia na matumizi mapana ya wazungumzaji wa asasi zinazoiunga mkono. Matumizi mapana ya lugha hujumuisha nguvu za kiuchumi, kisiasa na kijamii, pamoja na matumizi katika viwango mbalimbali vya elimu, idara za serikali kitaifa, kimaeneo na katika biashara, uchapishaji na matumizi mapana katika vyombo vya habari. Kwa msingi huo, mitazamo chanya na hasi kuhusu lugha ya kufundishia elimu ya juu nchini Tanzania inajengwa katika misingi inayochunguza matumizi yake mapana katika sekta mbalimbali, ikiwamo ya elimu. Sehemu hii inahusu taathira ya kihistoria na mitazamo ya kisiasa na kisera inayoifanya lugha ya Kiswahili kujengewa mashaka kama lugha ya kufundishia elimu ya juu.

\subsection{Kiswahili Katika Taathira ya Kihistoria na Mielekeo ya Utaifa}

Sababu za kihistoria kuhusu maendeleo ya Kiswahili katika nchi za Afrika Mashariki zimechangia kuwapo kwa mitazamo tofauti tofauti na mashaka ya Kiswahili kufundishia elimu ya juu. Kwa mfano, kati ya miaka ya 1920 na 1960, kabla ya nchi za 

Afrika Mashariki kupata uhuru, suala la usanifishaji wa lugha ya Kiswahili lilifanywa kwa ushirikiano wa nchi zote. Makubaliano ya pamoja kuhusu namna ya kuitumia lugha ya Kiswahili, kiisimu, ilitumiwa katika nchi zote za Afrika Mashariki (Msanjila na wenzake, 2009). Lakini, mara tu baada ya uhuru kila nchi ilifanya kazi ya usanifishaji peke yake. Ule muafaka uliokuwa ufikiwe kwa pamoja kwa matumizi ya msamiati na sarufi kwa ujumla, ukaanza kuteteleka. Huu ukawa mwanzo wa kusigana, hasa katika miundo na mitindo ya kiisimu, pamoja na kupungua kwa nguvu na kasi ya kukua na kukomaa kwa lugha ya Kiswahili kikanda.

Aidha, mielekeo ya utaifa iliyojitokeza baada ya uhuru wa nchi za Afrika Mashariki ilichangia kuathiri ukuzaji na uendelezaji wa lugha ya Kiswahili. Kamati ya lugha ya Kiswahili ya Afrika Mashariki iliyoundwa wakati wa Ukoloni ilihudumia nchi zote nne za Kenya, Tanganyika, Zanzibar na Uganda. Lakini, mara tu baada ya uhuru, kila nchi ilijiwekea utaratibu wake kuhusu namna ya kuikuza na kuiendeleza lugha ya Kiswahili. Kwa mfano, nchi ya Uganda haikupendezwa na lugha ya Kiswahili kutumika kama lugha rasmi humo nchini, wakati Tanzania na Kenya zilipendelea kuendelea kuitumia (Mukama, 1983). Licha ya serikali ya Uganda kukitangaza Kiswahili kuwa lugha ya taifa mnamo Agosti 7, 1973, lakini haikufundishwa katika shule za Uganda; na ilipopendekezwa lugha ya Kiswahili iongezwe katika orodha ya masomo katika jamii za Buganda, Bunyoro, Toro na Ankole ili kufuata mfano wa Tanzania na Kenya, ilipata upinzani mkali kutoka kwa Kabaka Daudi Chwa (Lugumba \& Ssekamwa, 1973). Kabaka alipinga kwa kusema:

Hapana haja hata kidogo kukitumia Kiswahili kiwe lugha rasmi ya Buganda kwani nina hakika jambo hili bila shaka litatupotezea cheo cha kabila letu na taifa katika makabila ya Afrika (Tuli, 1985, uk. 16).

Kabla ya miaka ya 1960, watawala nchini Uganda walihimiza matumizi ya lugha ya Kiingereza pekee katika nyanja zote za mawasiliano. Kuelekea miaka ya 1960, kama ilivyokuwa Tanzania na Kenya, Waingereza waligundua kwamba, Kiswahili kilitumiwa kama chombo muhimu cha kuwaunganisha wananchi katika juhudi zao za kupigania uhuru. Kwa hiyo, kwa vyovyote walidhamiria kuvunja vyombo vyote ambavyo vingeleta muungano wa Waafrika. Mtaalamu mmoja wa Uganda alisema:

Katika kuamka kwa Mwafrika baada ya vita vikuu yaingia akilini kuwa kuzuiliwa kwa matumizi ya Kiswahili kulikuwa na hila ya kupunguza muungano kati ya Waafrika. 
Juu ya hayo, bidii ya kuhimiza Kiingereza iliendelea hali ambayo ilitilia shaka kuwapo kwa lugha moja ya umma kungeleta kuangushwa kwa utawala wa kikoloni kwa haraka. Huku kutoungwa mkono na serikali, lugha ya Kiswahili itumiwayo na kila mtu kulikuwa na maana kuzuia muungano wa Waafrika baada ya vita usienee (Ndungo \& Mwai, 1991, uk. 23).

Kulingana na hali hiyo, kukawa hakuna umoja wa kikanda kwa mtazamo wa Uafrika Mashariki uliokuwa umeasisiwa enzi za ukoloni, hasa wakati wa usanifishaji wa lugha ya Kiswahili mapema miaka ya 1930. Msigano wa mielekeo ya kila taifa uliojibainisha mapema baada ya nchi za Afrika Mashariki kupata uhuru ulijenga mwanya wa kuidhoofisha lugha ya Kiswahili kikanda mapema miaka ya 1960 (Rono, 2001). Hali hiyo ilichangia lugha za kigeni katika nchi zote za Afrika Mashariki kuendelea kutumika katika utoaji wa elimu ya juu kama Tume ya Phelps-Stokes inavyoelezwa katika kipengele (c) kwamba, 'Lugha ya taifa la Ulaya linalotawala itumike kwenye masomo ya juu'. Aidha, lugha za kigeni zilihusishwa na maendeleo ya sayansi na teknolojia jambo linalofikiriwa kuwa kichocheo cha maendeleo ya jamii. Hali hiyo imeendelea kuleta ukinzani dhidi ya Kiswahili kutumika kufundishia elimu ya juu katika fani mbalimbali, hasa za sayansi na teknolojia.

\subsection{Kiswahili katika Mielekeo ya Kisiasa na Tabaka Tawala}

Kimsingi, mwelekeo wa kisiasa unaonesha msimamo uliochukuliwa na Waafrika walioshika mamlaka baada ya wakoloni wa kuendelea kupendelea kuyakumbatia mambo yote ya wakoloni, na kuyatumia kuzitawala nchi zao; na kuwa mstari wa mbele kuyapiga vita mambo ya jamii zao pengine kuliko walivyofanya wakoloni (Mekacha, 2000). Baadhi ya mambo yanayotukuzwa ni matumizi ya lugha za kigeni hata kufikia kuzibainisha nchi zao kwa majina ya lugha za kigeni walizorithi. Hali hii imezifanya lugha za kigeni ziendelee kuwa lugha za kufundishia katika ngazi mbalimbali za elimu, kwa msingi kwamba, ni lugha kubwa na zinazohusishwa na maendeleo ya sayansi na teknolojia (Mlama \& Matteru, 1977). Mwelekeo huo unakinzana na dhana muhimu za maendeleo ya jamii na uhuru wa taifa kwa kuwa unakwamisha maendeleo ya lugha za asili zinazooneshwa kuwa zinawawezesha wanajamii kueleza fikra zao za ndani na kulenga uelewa wa lugha unaowawezesha kuwa wabunifu na wagunduzi.

Mwelekeo wa kisiasa, hasa wa tabaka tawala umegharimu mataifa ya Kiafrika kutokana na sababu mbalimbali. Mojawapo ya sababu hizo ni kwamba, watawala wanahofu gharama kubwa za kuandaa vifaa vya kufundishia kwa kutumia lugha za 

asili na kufikiri kuwa ni nafuu kuendelea kutumia vifaa vya kufundishia vilivyokwishaandaliwa kwa lugha za wageni waliowatawala (Ansre, 1977; Qorro, 2000). Pia, wamekumbatiwa na dhana ya 'Dunia Kijiji' na hivyo wanajenga hoja ya wanafunzi kujua lugha za kimataifa ili waweze kuwasiliana na watu wa mataifa na makundi mengine (Edwards, 1982; Ryan, 1982; Ryanga, 2002). Mtazamo huu ndio unaozitazama lugha za kigeni katika misingi ya kupata maendeleo haraka ya kisayansi na kiteknolojia kwa kutumia lugha zilizokwishaendelea sana katika maeneo yaliyodokezwa. Mwelekeo huo ndio unaoitazama lugha ya Kiswahili kwa mashaka ya kufundishia elimu ya juu katika fani zote.

Kwa upande mwingine, watawala wa Kiafrika wameshindwa kutathmini matatizo ya kielimu yanayotokana na matumizi ya lugha za kigeni katika elimu. Utafiti unaonesha kuwa zaidi ya asilimia 95\% ya wanafunzi katika shule za sekondari nchini Tanzania hawaelewi Kiingereza (Campbell \& Qorro, 1979). Wanafunzi wengi wanashindwa kuelewa masomo yote yanayofundishwa kwa lugha hiyo. Kulingana na Shitemi (2001) na Qorro (2007) hii ina maana kwamba, madhumuni ya kuwapatia elimu hayatimii kwa kuwa baadhi ya walimu na wengi wa wanafunzi hawaielewi lugha inayotumika kufundishia (Kiingereza). Kwa maelezo mengine, kutumia lugha ya Kiingereza kufundishia kunapingana moja kwa moja na malengo na madhumuni mahususi ya elimu nchini Tanzania. Hali hii inaathiri ustawi wa taifa katika fani zake zote, ikiwamo elimu.

Mrikaria na wenzake (2007) wanaeleza miongoni mwa sababu za kushamiri kwa lugha ya Kiingereza katika nchi nyingi za Kiafrika na kutupilia mbali lugha za kiasili, ikiwamo ya Kiswahili. Baadhi yake ni kuogopa mabadiliko, ukosefu wa vitabu vya taaluma vinavyokidhi harakati za kisayansi na kiteknolojia, gharama, kuanguka kwa viwango vya elimu nchini kwa kuwa elimu inahusishwa na lugha, ikiwamo lugha ya Kiingereza. Kwa namna hali ilivyo sasa, si sahihi kufikiria kwamba kila penye asasi ya kielimu katika mataifa yote patazungumzwa Kiingereza (Mwangosi, 2007). Hii ni kwa sababu Watanzania wamekwenda China, Israel, Japani, Korea, Ufaransa, Ujerumani, Ureno na Urusi na sehemu nyingine ambazo hazitumii kabisa Kiingereza. Pengine ingekuwa bora kujifunza lugha kila inakozungumzwa na wazawa wake kuliko kama tunavyojifunza hapa Afrika lugha za kigeni huku tukipuuza na kushusha hadhi ya lugha za kiasili, kama ilivyo kwa upande wa Kiswahili hapa nchini. 
Kwa ujumla, mwelekeo wa kisiasa umegharimu mataifa ya Kiafrika kutokana na hofu isiyo ya msingi kuhusu gharama kubwa za kuandaa vifaa vya kufundishia kwa kutumia lugha za asili. Matokeo yake lugha za kiasili barani Afrika, ikiwamo ya Kiswahili, zimeendelea kunyimwa fursa pana ya kutumika kwa kufikiri kuwa ni nafuu kuendelea kutumia vifaa vya kufundishia vilivyokwishaandaliwa kwa lugha za wageni (wakoloni) waliowatawala.

\subsection{Kiswahili katika Mwelekeo wa Kasumba ya Kutawaliwa}

Kasumba ya kutawaliwa inajidhihirisha kwa baadhi ya makundi ya wananchi wa Kiafrika wanaopata mashaka ya kutumia lugha zao kuwa lugha za kufundishia kwa kupendelea kutumika kwa lugha za wakoloni baada ya ukoloni. Kasumba inaonesha mabadiliko ya fikra za mtu yanayosababishwa na elimu au utamaduni wa jamii unaomfanya kufuata mwenendo usio muafaka kwa utamaduni wake au utu wake wa asili (TUKI, 2004). Kasumba ndiyo chanzo cha wakoloni, pamoja na sababu nyingine, kuanza kuziweka kando lugha za asili, ingawa tume yao ya uchunguzi ilikwishapendekeza kwamba, lugha za asili ndizo zilizokuwa zinafaa kuwa lugha za kufundishia katika ngazi mbalimbali za elimu nchini (Tiffen, 1975). Badala ya kufanya jitihada za kuziendeleza na kuboresha misingi ya elimu kwa manufaa ya taifa, tabaka tawala linahangaika kuweka mikakati ya kuziimarisha lugha za kigeni na kupuuza jitihada za kuziimarisha lugha za asili ili zitumike katika kutolea maarifa mapana na ya kina. Hali hiyo ndiyo inayojidhihirisha kwa upande wa lugha ya Kiswahili ambayo bado inapewa nafasi duni kwa upande wa kufundishia elimu ya juu, licha ya kuendelezwa kwa takribani miaka 90 tangu iliposanifishwa miaka ya 1930.

\subsection{Sera ya Lugha}

Sera ni utaratibu wa kuendesha jambo kukidhi haja ya mamlaka inayohusika katika jamii mahususi. Sera ndiyo inayotamka malengo, misingi ya uteuzi wa lugha na michakato ya uendelezaji wake wa matumizi yake. Sera ya lugha ndiyo inayopaswa kuandaliwa na kukubaliwa kwanza kabla ya utaratibu mwingine kuanza kuzingatiwa (Bourhis, 1982). Kutokana na kurithi taratibu zilizoachwa na wakoloni, nchi nyingi za Kiafrika hazikuwahi kubuni sera zao za lugha. Hata zilipobuniwa, hazikuwahi kutekelezwa ipasavyo. Kwa mfano, nchini Tanzania imewahi kubuniwa Sera ya Utamaduni ambayo ilijumuisha masuala ya lugha mnamo mwaka 1997. Sera hiyo 

inataja kwamba, lugha ya kutumiwa kama lugha ya kufundishia katika ngazi zote za elimu itakuwa Kiswahili (Besha, 1999; Kiango, 1999). Hata hivyo, hadi leo hii haijatekelezwa kwa vitendo.

Sera ya Utamaduni hapa nchini inaeleza kwamba, lugha za asili zitahifadhiwa na kuwa chanzo cha taarifa zitakazotumika kuendeleza Kiswahili na ufundishaji wa lugha za kigeni utaimarishwa ili kuwawezesha wanajamiilugha kuzitumia vizuri (Edwards, 1985). Hata hivyo, Sera ya Utamaduni haijatekelezwa kwa vitendo; hata kama lugha ya Kiswahili ina sifa zinazotosheleza kufundishia elimu ya juu. Sera kama mwongozo wa mipango na utekelezaji ni muhimu kuandaliwa na kuidhinishwa na mamlaka kutumika kikamilifu. Kutokutekelezwa kwa Sera ya Utamaduni ni tatizo kwa upande wa lugha ya Kiswahili kupata nafasi pana ya kufundishia katika fani zote za kijamii, hasa za kisayansi na kiteknolojia.

\section{6.o Mapendekezo na Hitimisho}

Jambo la msingi linalopaswa kuzingatiwa katika uteuzi wa lugha ya kufundishia ni kufuata vigezo vya upangaji lugha na kuwekewa mikakati ya kuendelezwa ili kuwasilisha maarifa mapana na ya kina yanayopaswa kutolewa katika kila ngazi ya elimu inayohusika. Kipekee, sera ya lugha inapaswa kuandaliwa, kukubaliwa na kutekelezwa kwa vitendo. Aidha, suala la Kiswahili kuwa lugha ya kufundishia elimu ya juu hapa nchini linahitaji kutazamwa upya kwa kuzingatia muktadha wa mielekeo iliyodokezwa. Mabadiliko makubwa ya kiutendaji yakifanyika katika maeneo yaliyooneshwa na kutiliwa mashaka, hapana shaka kwamba, lugha za asili za Kiafrika, ikiwamo lugha ya Kiswahili zitapewa nafasi zinazostahili (Mhina, 1977). Kwa kufanya hivyo, mabadiliko ya kupendelea mambo ya ndani ya Afrika, hasa yenye tija yatakuwa yametimia.

\section{Marejeleo}

Ansre, G. (1977). 'Four Rationalizations for Maintaining the European Language in Education in Africa'. In Kiswahili, 47 (2), 55 - 61.

Besha, R. M. (1999). 'Nafasi ya Kiswahili katika Maendeleo ya Sayansi na Teknolojia. Katika Tumbo-Masabo Z. N., \& Chiduo, E. K. F (Wahariri). Kiswahili katika Elimu. Dar es Salaam: TUKI.

Bourhis, R. Y. (1982). 'Language Policy and Language Attitudes' katika Ryan, E \& B. 
Giles, H. (1982). Attitudes towards Language Variation. London: Edward Arnold.

Buliba, A., na Wenzake, (2006). Isimujamii kwa Wanafunzi wa Kiswahili. Nairobi: The Jomo Kenyatta Foundation.

Campbell, R., \& Qorro, M. (1979). The Language Crisis in Tanzania: The Myth of English versus Education. Dar es Salaam: Mkuki na Nyota Publishers.

Chachage, S. (2003). Kiswahili Katika Muktadha wa Utandawazi. Dar es Salaam: BAKITA.

Chiragdin, S., \& Mathias, M. (1977). Historia ya Kiswahili. Nairobi: Oxford University Press.

Edwards, J. R. (1982). Language, Society and Identity. Oxford: Basil Blackwell.

Edwards, J. R. (1985). 'Language Attitudes and Their Implications among English Speakers, katika: Ryan, E. B., \& Giles, H. (1982). Attitudes towards Language Variation, Social and Applied Contexts. London: Edward Arnold.

Jones, T. J. (1925). Education in Africa: A study of East Central and South Africa by the Second African Education Commission with the Internatinal Board. New York: Acadeic Press.

Kajenje, Y. (2007). 'Kiswahili, Utandawazi na Umoja wa Afrika' katika Insha za Jubilei 2005. Dar es Salaam: TUKI: 59-66.

Kalondero, K. (1983). 'Sura ya Kiswahili katika Zaire'. A Paper Read at the International Conference on the Standardazation of Kiswahili Terminologies. Dar es Salaam: Taasisi ya Uchunguzi wa Kiswahili, 43-49.

Kiango, J. G. (1999). ‘Uteuzi wa Lugha kwa Ajili ya Elimu' katika Z. N. Tumbo Masabo \& E. K. F. Chiduo (Wahariri), Kiswahili katika Elimu. Dar es Salaam: Taasisi ya Uchunguzi wa Kiswahili.

Lugumba, S. M. E., \& Ssekamwa, J. C. (1973). A History of Education in East Africa 1900-1973. Kampala: Government Printer.

Mammino, L. (2000). 'The Harmonisation and Standardization of Sango, the Official National Language of Central Africa'. In Prah, Kwesi Kwa (editor.) (2000) Rehabilitating African Languages. Cape Town: The Centre for Advanced Studies of African Society (CASAS).

Marani, J. (2007). ‘Hali ya Kiswahili Katika Vyuo Vikuu Nchini Kenya'. Dar es Salaam: CHAWAKAMA: 12-19.

Massamba, D. P. B. (1993). 'The Role of Language in Education and Research' katika Proceedings of African/ American/ Japanese Scholars Conference for Co- 

operation in Educational, Cultural and Environmental Spheres in Afria. Tokyo: 7281.

Mazrui, A. A. (1995). Swahili State and Society: The Political Economy of an African Language. Nairobi: East African Educational Publishers.

Mbaabu, I. (1991). Historia ya Usanifishaji wa Kiswahili. Nairobi: Longman.

Mbaabu, I. (2009). Historia ya Usanifishaji wa Kiswahili. Dar es Salaam: TUKI.

Mekacha, R. D. K, (2000). Isimujamii: Nadharia na Muktadha wa Kiswahili. Osaka: Osaka University of Foreign Studies.

Mhina, G. (1977). 'The Tanzania Experience in the Use of Kiswahili in Education. In Kiswahili.47 (2), 62-69.

Mlama, P., \& Matteru, M. (1977). 'Haja ya Kutumika Kiswahili Kufundishia Elimu ya Juu'.

Dar es Salaam: BAKITA.

Mrikaria, E. S., \& Wenzake (2007). (Wahariri). Isha za Jubilei 2005. Dar es Salaam: TUKI.

Msanjila, P. Y. (2009). Isimujamii Sekondari na Vyuo. Dar es Salaam: TUKI.

Mukama, R. (1983). 'Kukomaa, Hali na Hadhi ya Kiswahili Nchini Uganda'. A Paper Read at the International Conference on the Standardazation of Kiswahili Terminologies. Dar es Salaam: TUKI: 38-43.

Mwakisimba, D. (2007). 'Kiswahili, Utandawazi na Umoja wa Kiafrika' katika Insha za Jubilei 2005. Dar es Salaam: TUKI, 1-8.

Mwakyembe, F. (Novemba 13-19, 2003). 'Maendeleo ya Teknolojia si Kikwazo cha Lugha' RAI. Na 527: 18.

Mwangosi, A. W. (2007). 'Kiswahili, Utandawazi na Umoja wa Kiafrika' katika Insha za Jubilei 2005. Dar es Salaam: TUKI, 8-18.

Ndungo, C., \& Mwai, W. (1991). Historial Modern Development in Kiswahili. Nairobi: Nairobi University Press.

Ngugi wa Thiong'o, (1986). De-Colonizing the Mind. The Politics of Languagues in African Literature. Nairobi: E. A. E. P.

Ogechi, N. O. (2002). 'Mbinu za Mawasiliano kwa Kiswahili’. Moi University: Moi University Press.

Qorro, M. (2000). 'Mjadala wa Lugha ya Kufundishia: Sababu zilizotolewa Kupinga Matumizi ya Kiswahili kama Lugha ya Kufundishia' katika J. S. Mdee \& H. J. M. Mwansoko (Wahariri) Kiswahili 2000 Proceedings. Dar es Salaam: TUKI. 
Qorro, M. (2007). 'Matatizo ya Kufundisha kwa Lugha za Kigeni katika Nchi za Afrika: Kiingereza katika Shule za Sekondari Tanzania'. Dar es Salaam: Taasisi ya Uchunguzi wa Kiswahili.

Prah, K. K. (2003). 'Going Native: Language of Instruction for Education, Development and African Emancipation'. In B. Brock-Utne, Z. Depai \& M. Qorro (eds.) Language of Instruction in Tanzania (LOITASA). Dar es Salaam: E \& D Limited.

Rubagumya, C. M. (1986).'Planning in the Tanzania Education System: The Problems and Prospects' katika Journal of Multilingual and Multicultural Development, 7(4), 289-300.

Ryan, E. B. (1982). 'An Intergrative Perspective for the Study of Language' katika Attitudes Towards Language Variation. London: Edward Arnold.

Ryanga, S., et al. (2002). 'The African Union in the Wake of Globalization: The Forgetten Language Dimension'katika Kiswahili Juzuu 65.Dar es Salaam: TUKI, 115 .

Shitemi, N., et al. (2001). Kiswahili: A Tool for Development - The Multidisplinary Approach. Eldoret: Moi University Press.

Tiffen, B. (1975). 'English versus African Languages as a Medium of Education in African Primary Schools'. In Brown, G., \& Mervyn Hiskett (Eds.). Conflict and Harmony in Education in Tropical Africa, 4/2 \& 3: 129 - 147.

TUKI (2004). Kamusi ya Kiswahili Sanifu. Dar es Salaam: Taasisi ya Uchunguzi wa Kiswahili.

Tuli, R. S. K. (1985). Chimbuko la Kiswahili, Kukua na Kuenea katika Afrika ya Mashariki. Dar es Salaam: Utalii Exporters and Publications. 